\title{
Transposition of the lateral femoral cutaneous nerve
}

\author{
Amgad Hanna, MD \\ Department of Neurological Surgery, University of Wisconsin, Madison, Wisconsin
}

OBJECTIVE Meralgia paresthetica causes pain, burning, and loss of sensation in the anterolateral thigh. Surgical treatment traditionally involves neurolysis or neurectomy of the lateral femoral cutaneous nerve (LFCN). After studying and publishing data on the anatomical feasibility of LFCN transposition, the author presents here the first case series of patients who underwent LFCN transposition.

METHODS Nineteen patients with meralgia paresthetica were treated in the Department of Neurological Surgery at University of Wisconsin between 2011 and 2016; 4 patients underwent simple decompression, 5 deep decompression, and 10 medial transposition. Data were collected prospectively and analyzed retrospectively. No randomization was performed. The groups were compared in terms of pain scores (based on a numeric rating scale) and reoperation rates.

RESULTS The numeric rating scale scores dropped significantly in the deep-decompression $(p=0.148)$ and transposition $(p<0.0001)$ groups at both the 3-and 12-month follow-up. The reoperation rates were significantly lower in the deep-decompression and transposition groups $(p=0.0454)$ than in the medial transposition group.

CONCLUSIONS Both deep decompression and transposition of the LFCN provide better results than simple decompression. Medial transposition confers the advantage of mobilizing the nerve away from the anterior superior iliac spine, giving it a straighter and more relaxed course in a softer muscle bed.

https://thejns.org/doi/abs/10.3171/2017.8.JNS171120

KEYWORDS canal; lateral femoral cutaneous nerve; meralgia paresthetica; neurectomy; neurolysis; transposition; peripheral nerve

M ERALGIA paresthetica (Bernhardt-Roth syndrome) is manifested by pain, usually burning, in the anterolateral aspect of the thigh and is commonly associated with dysesthesia, allodynia, and decreased sensation in the lateral femoral cutaneous nerve (LFCN) territory. It is commonly observed in overweight males, after anterior hip surgery (direct trauma to the nerve), or prolonged surgery with the patient in a prone position (indirect trauma from compression). As previously shown, the LFCN runs in its own canal with a fascial plane completely surrounding the nerve. The most vulnerable spot is at the inguinal ligament where the canal is tightest and the nerve is angled, especially when it is close to or riding over the anterior superior iliac spine (ASIS). Diagnosis most often is made clinically. Nerve conduction studies are helpful only if the results are abnormal on the symptomatic side and normal on the other side. However, in obese patients, these results are hard to obtain. Ultrasound-guided nerve conduction studies can overcome this problem but can be uncomfortable for the patient. ${ }^{2}$ Pain relief after LFCN injections (including local anesthetics and long-acting steroids) aids in the diagnosis. Conservative measures include weight loss, injections (preferably ultrasound guided), and avoidance of tight belts or pants. Medications can include analgesic, antiinflammatory, and antiseizure agents (gabapentin). Surgery is offered when conservative measures fail and the pain is intractable. However, simple decompression is associated with a high failure rate, and neurectomy leaves the patient with an area of loss of sensation in the thigh and can be complicated by a painful neuroma.

ABBREVIATIONS ASIS = anterior superior iliac spine; BMI = body mass index; LFCN = lateral femoral cutaneous nerve; NRS = numeric rating scale.

SUBMITTED June 1, 2017. ACCEPTED August 8, 2017.

INCLUDE WHEN CITING Published online April 13, 2018; DOI: 10.3171/2017.8.JNS171120. 
Based on previously published work in which the anatomy of the LFCN canal was defined, ${ }^{7}$ a new LFCNtransposition technique was described, and special attention was paid to the fascia deep to the nerve (i.e., the deep layer of the LFCN canal). This technique offers $360^{\circ}$ decompression of the LFCN, mobilizes the nerve away from the ASIS, and straightens and shortens the course of the nerve. The anatomical feasibility of this technique has been determined already. ${ }^{8}$ This is the first series of patients in whom this technique was used.

\section{Methods}

Twenty-three patients with meralgia paresthetica were treated surgically between 2011 and 2016. Traumatic cases, mostly iatrogenic $(n=4)$, were excluded because of inherent damage to the nerve; only cases of spontaneous meralgia paresthetica were studied. This technique evolved chronologically from simple decompression $(\mathrm{n}=$ 4) to deep decompression $(n=5)$ to transposition $(n=10)$. Simple decompression involved releasing the deep fascia superficial to the LFCN and opening the inguinal ligament. For deep decompression, release of the deep fascia deep to the LFCN was added to open the LFCN canal completely and release the nerve entirely. For transposition, mobilization of the nerve approximately $2 \mathrm{~cm}$ medially away from the ASIS was added (Videos 1 and 2).

VIDEO 1. Video of left LFCN transposition. Copyright Amgad

Hanna. Published with permission. Click here to view.

VIDEO 2. Artistic rendition of right LFCN transposition. Copyright Amgad Hanna. Published with permission. Click here to view.

In 3 patients for whom the planned procedure was transposition, the nerve was too medial already, so deep decompression was performed instead. The numeric rating scale (NRS) scores (0-10) before, 3 months after, and 1 year after surgery were recorded. To avoid surgeon bias, the NRS scores were obtained from the nursing chart. Complications, including reoperation, were also recorded and compared among the 3 procedure groups. Data were collected prospectively and analyzed retrospectively.

Statistical analysis was performed using Statistical Analysis System (SAS Institute, Inc.) and Prism 6 (GraphPad Software). NRS scores were compared by using 1-way ANOVA to determine statistical differences between results, and if a result was deemed significant, Tukey post hoc analysis was used to test between specific groups. Reoperation rates were compared using the Fisher exact test. Quantitative data are presented as means \pm SEM. Differences were considered significant at $\mathrm{p}<0.05$.

This study was approved by the University of Wisconsin institutional review board.

\section{Results}

A total of 19 patients were treated surgically for spontaneous meralgia paresthetica between 2011 and 2016. There were 12 women and 7 men. Four patients underwent simple decompression, 5 deep decompression, and 10 LFCN transposition. Detailed demographic data are listed in Table 1. The mean patient age was 48.8 years (range $25-$ 72 years). No significant difference in patient ages among the 3 treatment groups was found $(\mathrm{p}=0.7314)$. The mean patient body mass index (BMI) was $30.9 \mathrm{~kg} / \mathrm{m}^{2}$ (range 22 $41.5 \mathrm{~kg} / \mathrm{m}^{2}$ ). There was no significant difference in patient BMIs among the 3 groups $(\mathrm{p}=0.3835)$. The mean preoperative NRS score was 6.6 (range 3-10). There also was no significant difference in preoperative NRS scores among the 3 groups $(p=0.9207)$.

In the simple-decompression group, the mean preoperative NRS score was 7 (range 5-8). This score dropped to 3.2 (range $0-8) 3$ months after surgery and to 1 (range $0-2) 1$ year after surgery. However, only $2(50 \%)$ patients made it to the 1-year follow-up; the other 2 patients underwent neurectomy for pain recurrence. The NRS scores before, 3 months after, and 12 months after surgery were not significantly different among patients treated with simple decompression ( $p=0.0867$ ) (Fig. 1A).

For the patients who underwent deep decompression, the NRS scores before, 3 months after, and 12 months after surgery were significantly different $(p=0.0148)$. The mean preoperative NRS score was 6.4 (range 3-10). The NRS scores dropped significantly to 1.6 (range 0-3) 3 months after surgery $(\mathrm{p}=0.0191)$ and to 2.2 (range $0-6) 1$ year after surgery $(\mathrm{p}=0.0392)$. All patients in this group had a 3-month and a 1-year follow-up visit (Fig. 1B).

In the LFCN-transposition group, all patients made it to the 3-month follow-up, but only $7(70 \%)$ had a 1-year follow-up visit (1 patient could not be reached, and for 2 patients, at the time of this writing, 1 year had not passed yet). The NRS scores before, 3 months after, and 12 months after surgery were significantly different $(\mathrm{p}<0.0001)$. The mean preoperative NRS score was 6.5 (range 4-10). The NRS scores dropped significantly to 1.5 (range 0-4) 3 months after surgery $(\mathrm{p}<0.0001)$ and to 1 (range $0-7) 1$ year after surgery $(\mathrm{p}<0.0001)$. Of the 7 patients who had a 1-year follow-up, 6 had an NRS score of 0 (Fig. 1C).

The mean reductions in NRS scores 3 months after surgery were 3.75 for simple decompression, 4.8 for deep decompression, and 5.0 for LFCN transposition. These reductions were not significantly different among the 3 treatment groups $(\mathrm{p}=0.7706)$ (Fig. 2A). One year after surgery, the mean NRS score reductions were 5.5 for simple decompression, 4.2 for deep decompression, and 6.0 for LFCN transposition. These reductions also were not significantly different among the 3 treatment groups $(\mathrm{p}=$ 0.6675) (Fig. 2B).

Because of pain recurrence, 2 (50\%) patients who underwent simple decompression required reoperation for nerve transection 6 months and 7 months later, respectively. One (20\%) patient who underwent deep decompression underwent reoperation to treat an infected hematoma. None of the 10 patients who underwent transposition underwent reoperation. This difference in reoperation rates was significantly different, with a p value of 0.0454 accounting for all reoperations (Fig. 3) and a p value of 0.0351 excluding the wound washout.

\section{Discussion}

The most common techniques used to treat meralgia paresthetica surgically are decompression (neurolysis) and neurectomy (transection) of the LFCN. Simple de- 
TABLE 1. Detailed patient data

\begin{tabular}{|c|c|c|c|c|c|c|c|c|c|}
\hline $\begin{array}{l}\text { Pt } \\
\text { No. }\end{array}$ & $\begin{array}{l}\text { Age } \\
\text { (yrs), } \\
\text { Sex }\end{array}$ & $\begin{array}{l}\mathrm{BMI} \\
(\mathrm{kg} / \\
\left.\mathrm{m}^{2}\right)\end{array}$ & Procedure & Side & $\begin{array}{l}\text { Preop } \\
\text { NRS } \\
\text { Score }\end{array}$ & Postop NRS Score(s) & Reop & $\begin{array}{l}\text { Postreop } \\
\text { NRS } \\
\text { Score(s) }\end{array}$ & Complication \\
\hline 1 & $40, F$ & 38 & S & $\mathrm{Lt}$ & 7 & 3 (at $1 \mathrm{mo}$ ), 5 (at $3 \mathrm{mos}$ ) & $\begin{array}{l}\text { Transect at } 6 \\
\text { mos }\end{array}$ & 0 (at $1 \mathrm{yr})$ & None \\
\hline 2 & $53, \mathrm{~F}$ & 28 & S & Lt & 5 & 0 (at 3 mos), 0 (at 1 yr) & None & NA & None \\
\hline 3 & $52, \mathrm{~F}$ & 32 & $S$ & $\mathrm{Lt}$ & 8 & 0 (at 3 mos), 2 (at 1 yr) & None & NA & None \\
\hline 4 & $32, \mathrm{M}$ & 30 & S & $\mathrm{Lt}$ & 8 & 6 (at $1 \mathrm{mo}$ ), 8 (at 3 mos) & $\begin{array}{l}\text { Transect at } 7 \\
\text { mos }\end{array}$ & $\begin{array}{r}3 \text { (at } 1 \mathrm{mo}), 0 \\
\text { (at } 3 \mathrm{mos})\end{array}$ & $\begin{array}{l}\text { Rt C-7 radiculopathy after } \\
\text { transect }\end{array}$ \\
\hline 5 & $45, \mathrm{~F}$ & 32.5 & D & Rt & 7 & 3 (at 3 mos), 0 (at 1 yr) & None & NA & None \\
\hline 6 & $51, \mathrm{~F}$ & 26 & D & Lt & 4 & 2 (at 3 mos), 6 (at 1 yr) & None & NA & $\begin{array}{l}\text { Offered revision w/ tran- } \\
\text { sect, but pt declined }\end{array}$ \\
\hline 7 & $25, \mathrm{~F}$ & 28 & $\mathrm{~T}$ & $\mathrm{Lt}$ & 6 & 0 (at 3 mos), 0 (at 1 yr) & None & NA & None \\
\hline 8 & $47, \mathrm{M}$ & 31.5 & $\mathrm{~T}$ & $\mathrm{Lt}$ & 4 & 2 (at 3 mos) & None & NA & None \\
\hline 9 & $72, \mathrm{~F}$ & 31.5 & $\mathrm{~T}$ & $\mathrm{Lt}$ & 8 & 2 (at 3 mos), 0 (at 1 yr) & None & NA & Wound infection, $A B X$ only \\
\hline 10 & $46, M$ & 31.2 & $\mathrm{~T}$ & Rt & 4 & 0 (at $1 \mathrm{mo}$ ), 0 (at $1 \mathrm{yr}$ ) & None & NA & None \\
\hline 11 & $57, \mathrm{M}$ & 29.8 & $\mathrm{~T}$ & Rt & 10 & $\begin{array}{l}0 \text { (at } 5 \text { days), 3-7 (at } 4 \text { mos), } \\
7 \text { (at } 1 \text { yr, gets injections } \\
\text { every } 6 \text { mos) }\end{array}$ & None & NA & Hematoma, tapped once \\
\hline 12 & $57, \mathrm{~F}$ & 32.7 & $\mathrm{~T}$ & Rt & 9 & 0 (at $1 \mathrm{yr})$ & None & NA & None \\
\hline 13 & $46, \mathrm{M}$ & 22 & $\mathrm{~T}$ & Rt & 8 & 2 (at 3 mos), 6 (at 6 mos) & None & NA & None \\
\hline 14 & $70, \mathrm{~F}$ & 33.6 & $\begin{array}{l}\mathrm{D} \text { (too medial to } \\
\text { transpose) }\end{array}$ & Rt & 10 & 0 (at $1 \mathrm{yr})$ & Wound washout & NA & Infected hematoma \\
\hline 15 & $51, \mathrm{M}$ & 30.5 & $\mathrm{~T}$ & Lt & 4 & 0 (at 8 mos) & None & NA & None \\
\hline 16 & $54, \mathrm{~F}$ & 25 & $\begin{array}{l}\mathrm{D} \text { (too medial to } \\
\text { transpose) }\end{array}$ & $\mathrm{Rt}$ & 3 & $\begin{array}{l}0 \text { (at } 1 \mathrm{mo}), 3 \text { (at } 3 \mathrm{mos}), 2 \\
\quad(\text { at } 1 \mathrm{yr})\end{array}$ & None & NA & None \\
\hline 17 & $27, \mathrm{~F}$ & 25 & $\begin{array}{l}\mathrm{D} \text { (too medial to } \\
\text { transpose; moved } \\
\text { by only } 1 \mathrm{~cm} \text { ) }\end{array}$ & Rt & 8 & $\begin{array}{l}0 \text { (at } 2 \text { wks), } 0 \text { thigh, } 8 \text { groin } \\
\text { (at } 3 \text { mos) (seroma), } 3 \text { (at } \\
1 \text { yr, mostly groin) }\end{array}$ & None & NA & $\begin{array}{l}\text { Groin seroma tapped } \\
\text { twice }\end{array}$ \\
\hline 18 & $60, M$ & 41.5 & $\mathrm{~T}$ & Rt & 4 & 1 (at $3 \mathrm{mos}$ ) & None & NA & None \\
\hline 19 & $42, \mathrm{~F}$ & 35.5 & $\mathrm{~T}$ & Rt & 8 & 4 (at 3 mos) & None & NA & None \\
\hline
\end{tabular}

$\mathrm{ABX}=$ antiobiotics; $\mathrm{D}=$ deep decompression; $\mathrm{NA}=$ not applicable; $\mathrm{pt}=$ patient $\mathrm{S}=$ simple decompression; $\mathrm{T}=\mathrm{LFCN}$ transposition; transect = transection.

compression has a high failure rate $(40 \%-70 \%),{ }^{4,14}$ so some surgeons opt for neurectomy as the primary procedure. ${ }^{3,4,6,14,15}$ Neurectomy leaves the patient with an area of permanent sensory loss in the thigh, and if a neuroma develops at the cut end of the nerve, it can be painful. ${ }^{5}$ I believe that one of the reasons that simple decompression fails is the development of scar tissue; the nerve becomes retethered in close proximity to the ASIS, and symptoms recur (Fig. 4). ${ }^{8}$ By transposing the nerve medially away from the bone, deeply in a softer muscle bed, and straightening its course, the nerve is in a better location even if scar tissue develops and is not tethered. Previous attempts at transposition have been made, but to my knowledge, no such case series have been presented, and the techniques were never popularized. ${ }^{1,9,10}$ Part of the problem is probably the lack of understanding of the anatomy of the fascial planes around the nerve. For years, surgeons have neglected decompressing the fascia deep to the nerve. They tend to decompress what is superficial to nerves and not pay attention to fascial planes deep to them. The current literature does not separate superficial and deep decompression, which makes it difficult to interpret the results.
These procedures are usually lumped under the term "neurolysis" or "decompression." However, the term "neurolysis" is inaccurate because it can mean literally "nerve ablation." In at least 3 case series, very good results with neurolysis alone were described; however, the detailed methods clearly defined deep decompression. Nahabedian and Dellon ${ }^{11}$ reported a 96\% success rate with excision of all overlying and underlying fascia. Son et al. ${ }^{13}$ and Siu and Chandran ${ }^{12}$ reported $100 \%$ and $93 \%$ success rates, respectively, with neurolysis, but they always cut the sling of fascia posterior to the nerve at the level of the inguinal ligament. The patients in my case series experienced better results with deep decompression with or without transposition than with merely superficial decompression. The benefit from transposition could be attributed at least partially to the $360^{\circ}$ decompression. Despite the anatomical variability in the course of the LFCN and its relationship to the ASIS, in most symptomatic patients, the nerve is very close to or riding over the ASIS. ${ }^{9,11}$ Therefore, mobilizing the nerve away from the bone, as described here in this new technique, makes sense. To avoid postoperative hematoma, which probably correlates with the more- 

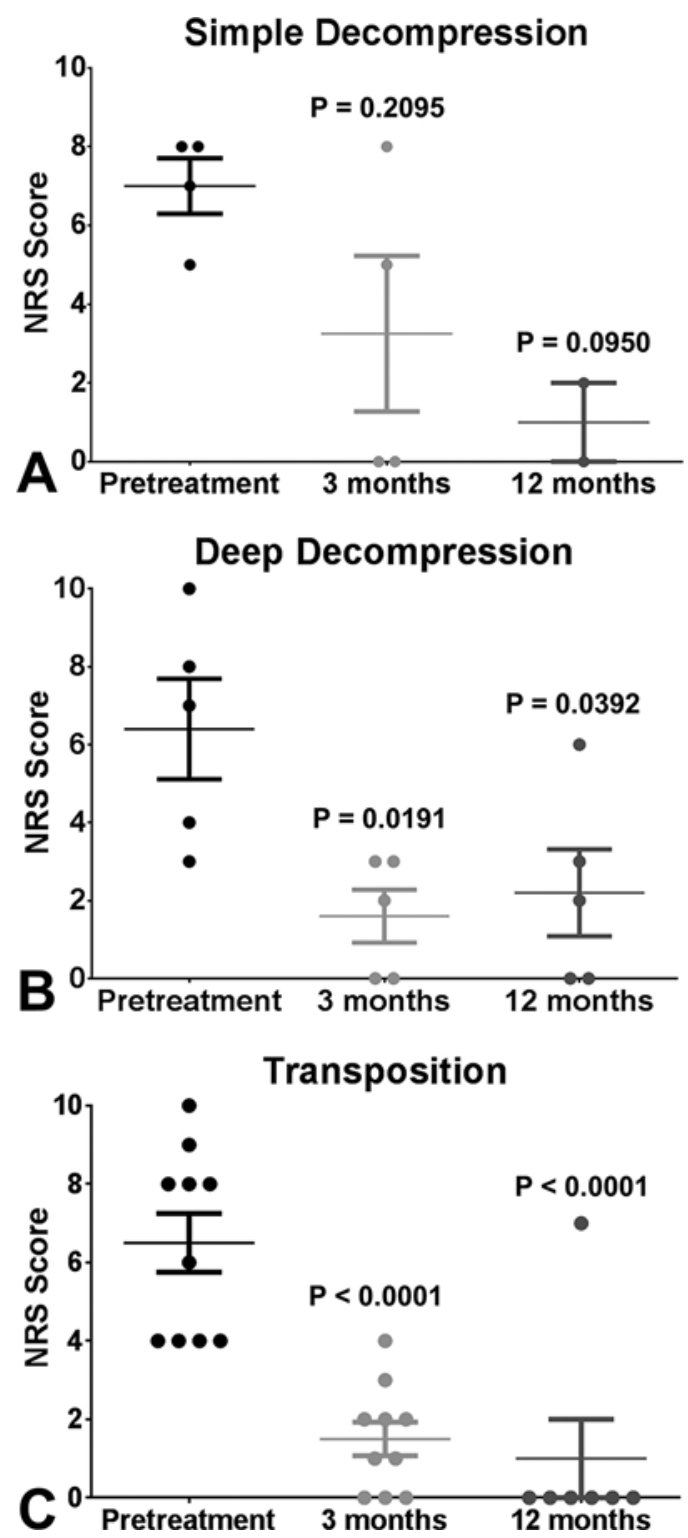

FIG. 1. Comparison of patient NRS scores before and 3 and 12 months after surgery (all 3 treatment types). A: No significant difference in NRS scores after simple decompression was found ( $p=0.0867,1$-way ANOVA). B: NRS scores of patients who underwent deep decompression changed significantly after surgery $(p=0.0148,1$-way ANOVA); scores were significantly lower 3 and 12 months after surgery than they were before surgery $(p<0.05$, Tukey post hoc test). C: NRS scores of patients who underwent LFCN transposition changed significantly after surgery ( $p<0.0001$, 1-way ANOVA); scores were significantly lower 3 and 12 months after surgery than they were before surgery $(p<0.0001$, Tukey post hoc test). Six of the 7 patients who underwent LFCN transposition were experiencing no pain 12 months after surgery and gave an NRS score of 0 . Error bars represent \pm the SEM.

extensive dissection, it is my practice now to leave a small drain for 24 hours after surgery.

This technique is not a one-size-fits-all approach. Sometimes the nerve is already too medial and straight in its course, rendering transposition not feasible; in such a case, deep decompression should be enough (patients 14, 16, and 17).

\section{Months Postoperatively}

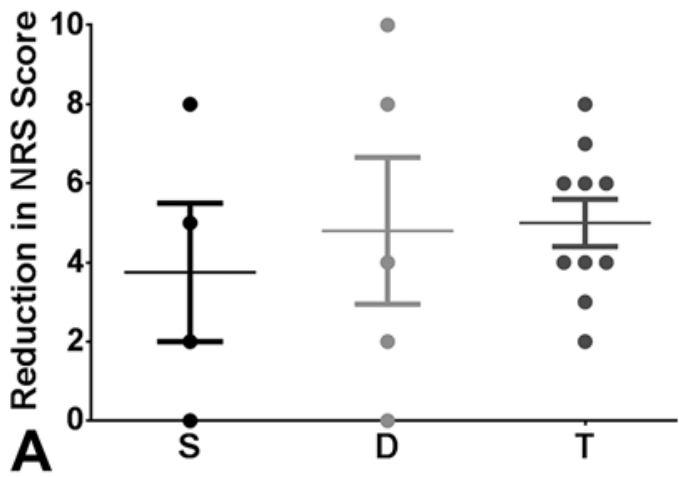

12 Months Postoperatively

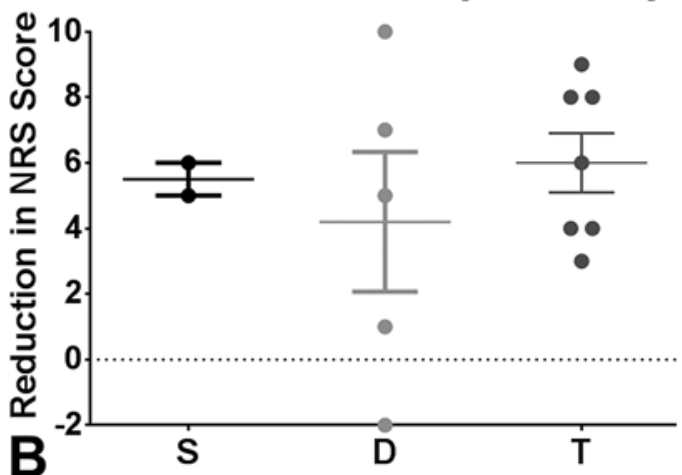

FIG. 2. Plots showing the reductions in NRS scores after surgery (all 3 treatment types). A: Three months after surgery, no significant difference in reduction in NRS scores among the 3 treatments was found $(p=$ 0.7706, 1-way ANOVA). B: Twelve months after surgery, no significant difference between the 3 treatments in reducing the NRS score was found ( $p=0.6675,1$-way ANOVA). Error bars represent \pm the SEM.

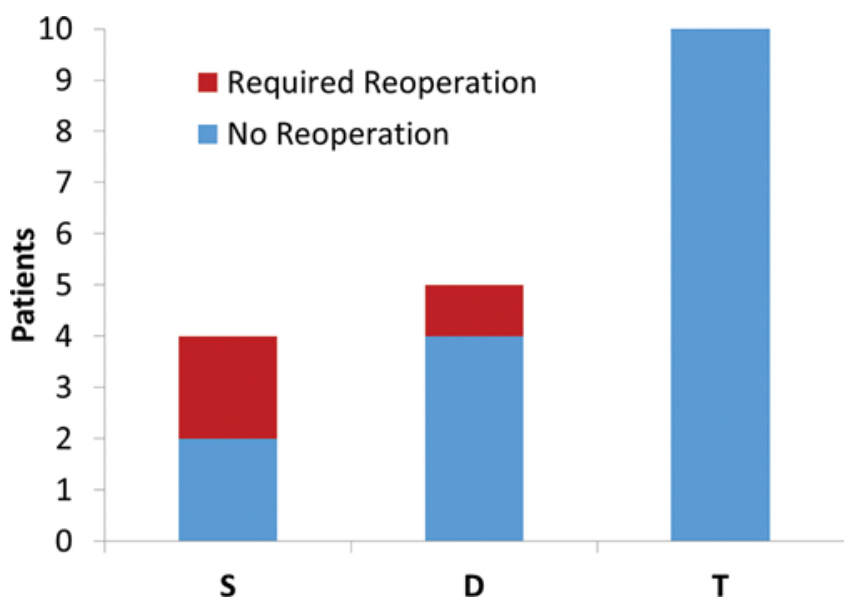

FIG. 3. Stacked-column chart showing the number of patients who required a second operation. When nerve transection or wound washout was considered as a reoperation, a significant difference between the treatments was found ( $p=0.0454$, Fisher exact test). The results were also significantly different when only nerve transection was considered a reoperation ( $p=0.0351$, Fisher exact test). None of the patients who underwent transposition needed a second operation. $S=$ simple decompression; $\mathrm{D}=$ deep decompression; $\mathrm{T}=\mathrm{LFCN}$ transposition. 


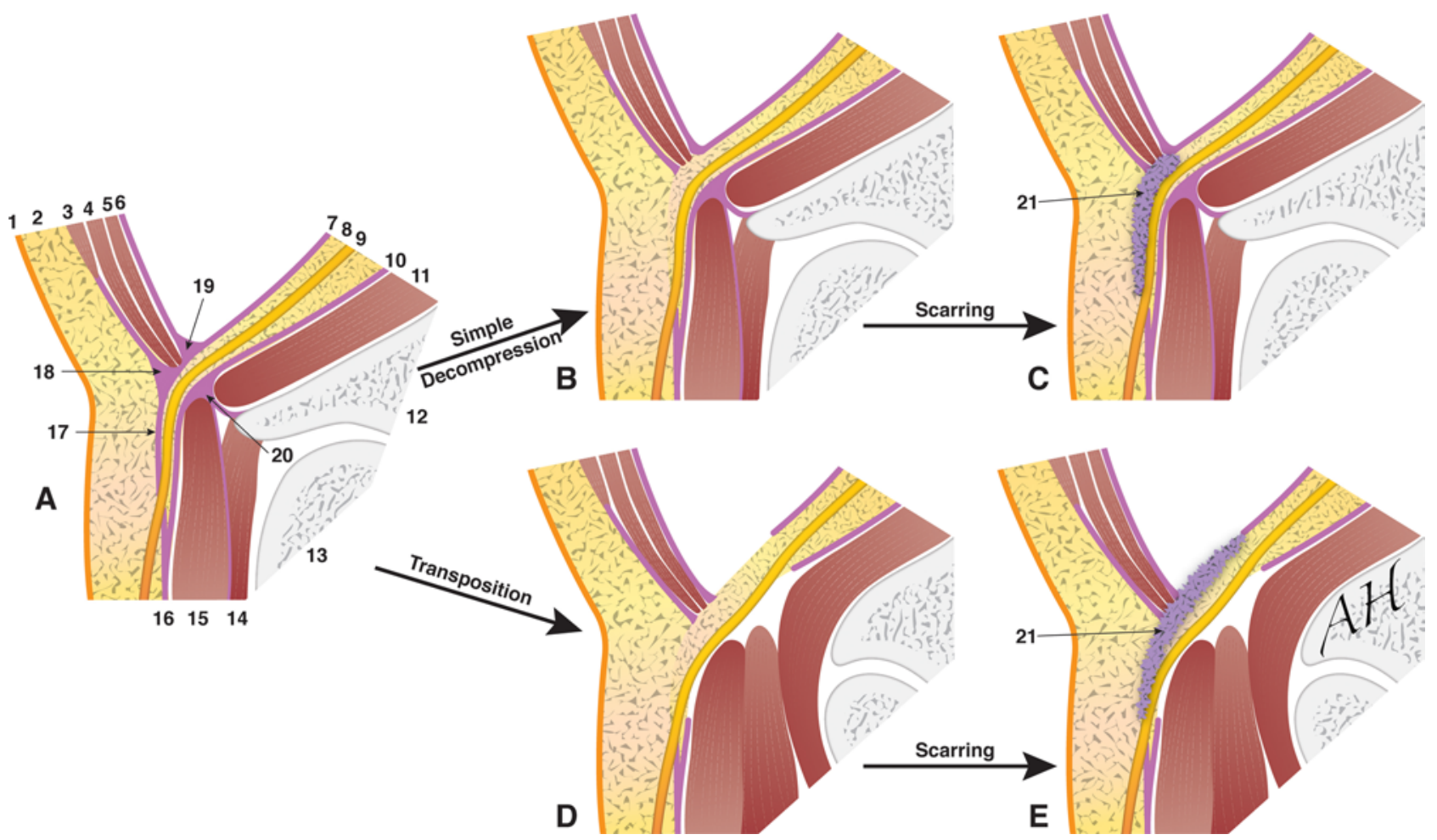

FIG. 4. A: Diagrammatic representation of the LFCN canal in an oblique sagittal view at the level of the ASIS. $1=$ skin; $2=$ subcutaneous tissue; 3 = external oblique muscle and aponeurosis; 4 = internal oblique muscle; 5 = transversus abdominis; 6 $=$ transversalis fascia; $7=$ fascia iliaca superficial lamina; $8=$ retroperitoneal fat; $9=\mathrm{LFCN} ; 10=$ fascia iliaca deep lamina; $11=$ iliacus; 12 = iliac bone; 13 = femur; 14 = rectus femoris; 15 = sartorius; 16 = fascia lata; $17=$ LFCN canal; $18=$ inguinal ligament; $19=$ iliopubic tract; 20 = thickening of the fascia deep to the LFCN, here by the tendinous origin of the sartorius (can also be a slip from the inguinal ligament or periosteum if the nerve is riding over the ASIS or iliac crest). Reproduced from Hanna: The lateral femoral cutaneous nerve canal. J Neurosurg 126:972-978, 2017. Copyright Amgad Hanna. Published with permission. B: After simple decompression, the deep fascia superficial to the nerve is released, as is the inguinal ligament. C: When scar tissue (21) develops, the nerve is retethered into a position very similar to that in the preoperative state (shown in A). D: With transposition, all components of the LFCN canal are opened superficial and deep to the nerve, and then the LFCN is mobilized approximately 2 $\mathrm{cm}$ medially. E: After transposition, the nerve acquires a much straighter and relaxed course, has a softer muscular bed, and is further away from the ASIS, even after scar tissue (21) develops. B-E: Reproduced from Hanna: Lateral femoral cutaneous nerve transposition: renaissance of an old concept in the light of new anatomy. Clin Anat 30:409-412, 2017. Copyright Wiley Periodicals. Published with permission.

Weaknesses in this study exist. First, the number of patients was small, which is probably why no significance was found when comparing the reduction in the NRS scores in the 3 groups; however, the rate of reoperation in the transposition group was overwhelmingly better than the rates in the other groups. The NRS is a good scale for comparisons within patient groups but not across them, which could further explain the lack of statistical significance found in the intergroup comparison and the significant differences found in the intragroup analysis. Second, although the data were collected prospectively, the patients were not randomly assigned to their treatment. The treatment was selected chronologically as I modified my technique. Therefore, some of the treatment results could be accounted for by the gaining of more experience. Third, for none of the groups was transection the primary treatment. Because permanent sensory loss occurs with transection, I do not believe it should be considered the primary treatment but, rather, saved for recurrence. Treating nerve entrapment by cutting the nerve is counterintuitive and is almost equivalent to euthanasia for treating cancer pain.

\section{Conclusions}

The results of this study provide evidence for improvement after surgical treatment for meralgia paresthetica when deep decompression is added to superficial decompression. Benefit also might exist for medial transposition of the LFCN after deep decompression. A prospective study to compare deep decompression and transposition might be of value in the future. As more centers explore this technique, we should continue gathering data to compare larger numbers of patients.

\section{Acknowledgments}

I thank Qianqian Zhao and Daniel Hellenbrand for their help with the statistics and Mark Ehlers and Evan Schultz for their assistance with the videos. 


\section{References}

1. Aldrich EF, van den Heever CM: Suprainguinal ligament approach for surgical treatment of meralgia paresthetica. Technical note. J Neurosurg 70:492-494, 1989

2. Boon AJ, Bailey PW, Smith J, Sorenson EJ, Harper CM, Hurdle MF: Utility of ultrasound-guided surface electrode placement in lateral femoral cutaneous nerve conduction studies. Muscle Nerve 44:525-530, 2011

3. de Ruiter GC, Kloet A: Comparison of effectiveness of different surgical treatments for meralgia paresthetica: Results of a prospective observational study and protocol for a randomized controlled trial. Clin Neurol Neurosurg 134:7-11, 2015

4. de Ruiter GC, Wurzer JA, Kloet A: Decision making in the surgical treatment of meralgia paresthetica: neurolysis versus neurectomy. Acta Neurochir (Wien) 154:1765-1772, 2012

5. de Ruiter GC, Wurzer JA, Kloet A: Recurrence of meralgia paresthetica years after a neurexeresis procedure: a case report. Br J Neurosurg 29:885-887, 2015

6. Emamhadi M: Surgery for meralgia paresthetica: neurolysis versus nerve resection. Turk Neurosurg 22:758-762, 2012

7. Hanna A: The lateral femoral cutaneous nerve canal. J Neurosurg 126:972-978, 2017

8. Hanna AS: Lateral femoral cutaneous nerve transposition: Renaissance of an old concept in the light of new anatomy. Clin Anat 30:409-412, 2017

9. Keegan JJ, Holyoke EA: Meralgia paresthetica. An anatomical and surgical study. J Neurosurg 19:341-345, 1962

10. Lee FC: An osteoplastic neurolysis operation for the cure of meralgia paresthetica. Ann Surg 113:85-94, 1941

11. Nahabedian MY, Dellon AL: Meralgia paresthetica: etiology, diagnosis, and outcome of surgical decompression. Ann Plast Surg 35:590-594, 1995
12. Siu TL, Chandran KN: Neurolysis for meralgia paresthetica: an operative series of 45 cases. Surg Neurol 63:19-23, 2005

13. Son BC, Kim DR, Kim IS, Hong JT, Sung JH, Lee SW: Neurolysis for meralgia paresthetica. J Korean Neurosurg Soc 51:363-366, 2012

14. van Eerten PV, Polder TW, Broere CA: Operative treatment of meralgia paresthetica: transection versus neurolysis. Neurosurgery 37:63-65, 1995

15. Williams PH, Trzil KP: Management of meralgia paresthetica. J Neurosurg 74:76-80, 1991

\section{Disclosures}

The author reports no conflict of interest concerning the materials or methods used in this study or the findings specified in this paper.

\section{Supplemental Information \\ Videos}

Video 1. https://vimeo.com/242945412.

Video 2. https://vimeo.com/242945519.

\section{Previous Presentations}

This work was presented in part as an electronic poster at the American Society of Peripheral Nerves meeting, Kauai, Hawaii, January 10, 2014, and the American Association of Neurological Surgeons meeting, San Francisco, California, April 5, 2014.

\section{Correspondence}

Amgad Hanna: University of Wisconsin, Madison, WI. ah2904@ yahoo.com. 